\title{
Urgent carotid endarterectomy in carotid artery stenosis with a pedunculated thrombus: a case report
}

\author{
Muhammet Akyüz, Onur Işık, Mehmet Fatih Ayık, Yüksel Atay \\ Received: June 11, 2014 Accepted: October 25, 2014 Published online: February 10, 2015
}

Intraluminal thrombus in the context of carotid artery stenosis is rarely encountered. The prevention of major disabling stroke is one of the foremost goals of the urgent treatment of carotid lesions with an intraluminal thrombus. Urgent carotid endarterectomy following non-disabling stroke or crescendo transient ischemic attacks is a safe and feasible procedure with favorable outcomes. Endovascular therapy may not be suitable in selected cases. Carotid artery stenosis with a pedunculated thrombus, as in the present case, has a higher risk of embolization than other intraluminal thrombi. Herein, we describe a case with recurrent episodes of amaurosis fugax, who underwent urgent carotid surgery due to a pedunculated thrombus.

Keywords: Carotid endarterectomy; endarterectomy; pedunculated thrombus.

Intraluminal carotid artery thrombus (ICAT) is an infrequent finding in patients presenting with transient ischemic attack (TIA) or ischemic stroke. ${ }^{[1]} \mathrm{It}$ can be found both in the presence or absence of carotid artery stenosis. ${ }^{[2]}$ An intraluminal thrombus may occur secondary to the extension of intraplaque hemorrhage and may produce significant morbidity and mortality, in case of proceeding to thrombotic occlusion or embolic events.

In the North American Symptomatic Carotid Endarterectomy Trial (NASCET), the risk of perioperative stroke and death doubled in the patients with ICAT who underwent carotid endarterectomy (CEA) compared to the patients without ICAT who underwent $\mathrm{CEA}$ (12\% vs. $6 \%$, respectively). ${ }^{[3]}$ Therefore, the optimal management of the patients with symptomatic ICAT still remains poorly defined. Treatment options include medical management utilizing anticoagulation with or without antiplatelet drugs ${ }^{[3]}$ early carotid revascularization with CEA or carotid artery stenting (CAS). ${ }^{[1]}$

Percutaneous transluminal angioplasty (PTA) and CAS have been increasingly employed for the treatment of high-risk patients with carotid artery stenosis, ${ }^{[4]}$ however, surgical treatment for the presence of carotid artery stenosis with pedunculated thrombus is a proper option. The major concern for carotid endarterectomy procedures with a pedunculated thrombus was the timing of surgery. Urgent surgical treatment was advocated for patients with an intraluminal thrombus to prevent neurologic deterioration. The present paper describes a case of carotid artery stenosis with a pedunculated thrombus in the light of literature data.

\section{CASE REPORT}

A 68-year-old woman with a history of hypercholesterolemia presented with a one-month history of TIA with amaurosis fugax. She was hospitalized in the neurology department. She had three to four attacks within one month, each lasting for 30 to 40 minutes. Physical examination revealed a blood pressure of 140/90 $\mathrm{mmHg}$. A right carotid bruit was noted and the neurological examination findings were normal. A computed tomography (CT) brain scanning was normal and carotid duplex scanning showed significant stenosis of the right internal carotid artery (ICA). The left carotid artery had normal flow patterns. Following a diagnosis of carotid artery stenosis, the patient received aspirin $(100 \mathrm{mg} / \mathrm{day})$ and clopidogrel (75 mg/day). Neurological status was stable and elective CAS was planned. Computed tomography angiography was planned for the evaluation of cerebral parenchyma and supra-aortic vessel before elective CAS. However, the patient was diagnosed with

Department of Cardiovascular Surgery, Medical Faculty of Ege University, İzmir, Turkey

Corresponding author: Muhammet Akyüz, M.D. Ege Üniversitesi Tıp Fakültesi Kalp ve Damar Cerrahisi Anabilim Dal, 35100 Bornova, İzmir, Turkey.

Tel: +90 232 - 441343 e-mail: drmak100@gmail.com 

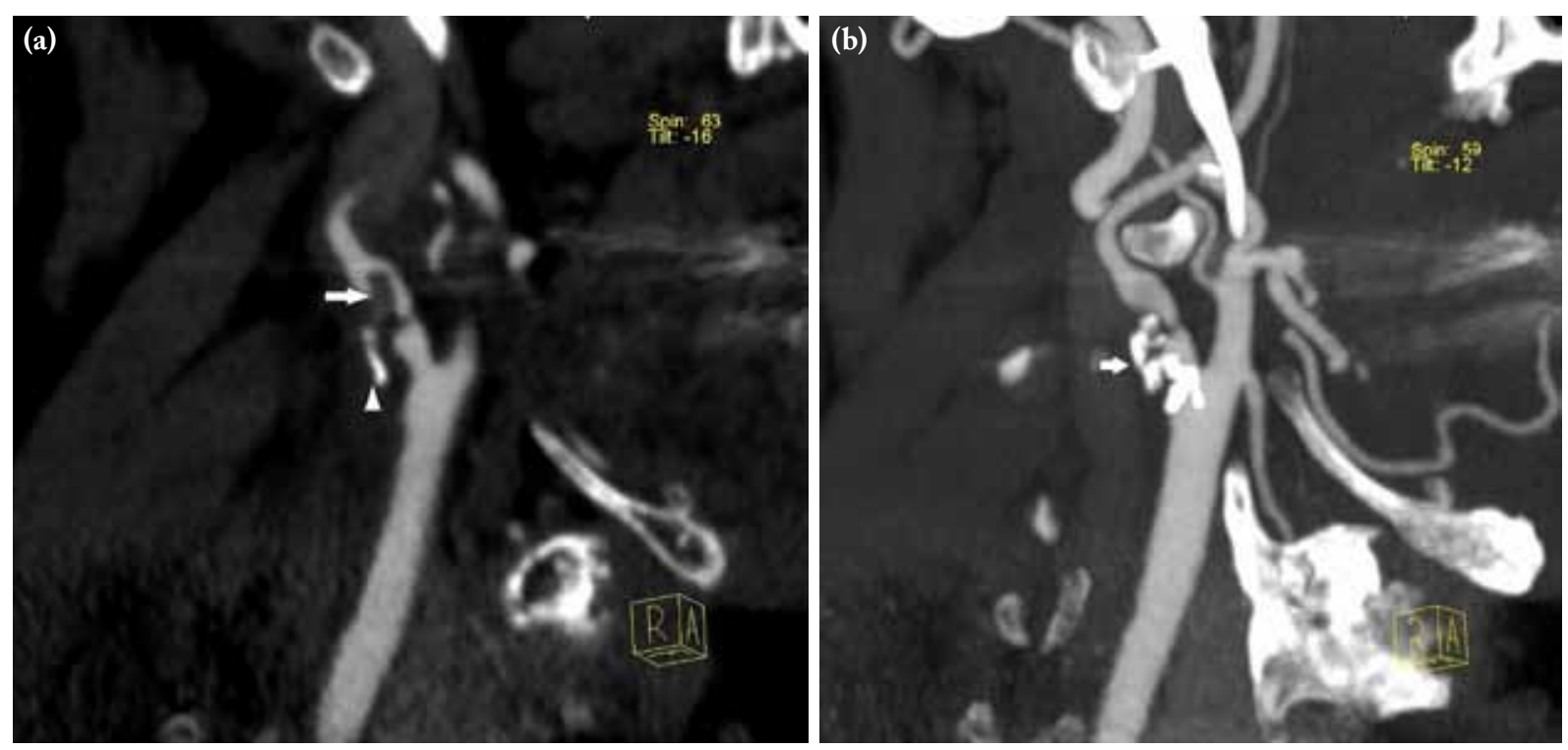

Figure 1. (a) Computed tomography angiography showing a right severe carotid bulb stenosis with deep plaque ulceration (arrow, pedunculated thrombus; arrowhead, bulb plaque ulcer). (b) A markedly right carotid artery stenosis (approximately 90\%, arrow).

intraluminal pedunculated thrombus by diagnostic CT angiography (Figure 1). Thus, urgent right CEA was planned and performed to prevent an ongoing distal embolism or acute occlusion. Under general anesthesia, CEA was performed. Cerebral oximetry using near-infrared spectroscopy (NIRS) was used to monitor cerebral oxygenation during operation. Regional cerebral oxygen saturation $\left(\mathrm{rSO}_{2}\right)$ was monitored continuously throughout the procedure. We based on the typical range of $\mathrm{rSO}_{2}$ is $55-80 \%$ and absolute $\mathrm{rSO}_{2}$-values $<50 \%$ or a $20 \%$ drop from individual $\mathrm{rSO}_{2}$ baseline are commonly considered as an intervention trigger.

During surgical exposure, manipulation of the carotid artery was avoided due to the presence of a pedunculated thrombus. After systemicheparinization, the common, internal, and external carotid arteries (CCA, ICA, ECA) were clamped. Conventional CEA was performed through a longitudinal arteriotomy from the CCA bifurcation to the ICA on the anterior surface of the artery. In the arteriotomy, pedunculated thrombus was noted association with the plaque in ICA and the thrombus was removed from the proximal end of the ICA (Figure 2). Endarterectomy was carried out following a careful identification of the cleavage plane. The distal intima was secured to prevent intimal dissection with 6-0 prolene sutures. The arteriotomy site was closed primarily without using the shunt due to distal ICA systolic tension over $50 \mathrm{mmHg}$ pressured after cross-clamping and the $\mathrm{rSO}_{2}$ did not drop to more than $12 \%$ of the preclamping value during clamping.

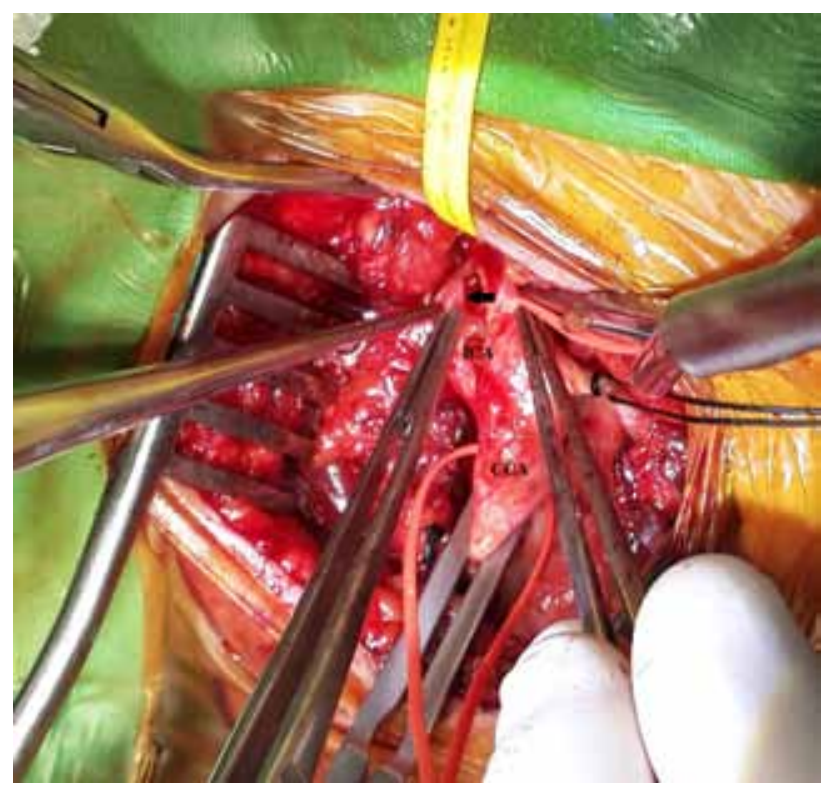

Figure 2. Carotid endarterectomy in progress. Plaque can be seen in the lumen of the common carotid artery (CCA) and the internal carotid artery (ICA). The pedunculated thrombus in the lumen of the ICA is also identified with a forceps (arrow). 
The patient was discharged three days later with combined clopidogrel-aspirin therapy and antihypertensive medication. At the follow-up, the duplex showed normal flow in the carotid arteries.

\section{DISCUSSION}

The optimal strategy for the treatment of carotid artery stenosis with intraluminal thrombus remains controversial. This condition is rarely encountered. Buchan et al. ${ }^{[1]}$ reported that stroke and occlusion did not occur in 14 patients with ICA stenosis and concomitant thrombus treated medically (e.g., heparin and warfarin). In contrast, Walters et al. ${ }^{[5]}$ advocated emergent surgical treatment for patients with an intraluminal thrombus to prevent neurologic deterioration. However, there are relatively few reports describing outcomes of PTA and CAS for the treatment of carotid artery stenosis with an intraluminal thrombus. ${ }^{[6]}$

In the NASCET study, Villarreal et al. ${ }^{[3]}$ reported that the 30-day risk of stroke or death for patients with an intraluminal thrombus was $12.0 \%$ and $10.7 \%$ for patients treated with surgery or medical therapy, respectively. Urgent carotid surgery versus percutaneous approach seems to be the most feasible option as in the present case. On the other hand, there are no clear guideline recommendations for the management of the patients with acute stroke or highrisk of acute stroke.

There are three groups of symptomatic patients presenting with possible indications for emergent carotid endarterectomy, including crescendo transient ischemic attacks, fluctuating neurological deficit, and acute stroke in evolution. ${ }^{[2]}$ The goal of surgery is to improve the existing perfusion by removing the thrombus or the source of emboli and to protect against further stroke. Furthermore, early surgery before a possible acute stroke may decrease the higher risk of intracranial bleeding and embolization due to treatment options as thrombolysis or endovascular intervention.

The American Heart Association's Guidelines for prevention of stroke in patients with ischemic stroke or transient ischemic attack recommend surgery within two weeks, when CEA is indicated for patients with TIA or stroke (Class IIa, level of evidence B). ${ }^{[7]}$ The risk of treatment options in relation to the clinical condition and type of stenosis may decrease with appropriate timing of treatment. If the patient, as in the present case, is diagnosed with carotid artery stenosis with a pedunculated thrombus, surgery should be planned immediately. The authors of the current study performed CEA, as soon as the diagnosis was made by CT.

Although CAS was feasible in the presence of an intraluminal thrombus with or without carotid artery stenosis, it has the higher risk of procedure-related thromboembolic events.

In conclusion, the authors believe that urgent endarterectomy compared to CAS remains the best option in patients of carotid artery stenosis with a pedunculated thrombus.

\section{Declaration of conflicting interests}

The authors declared no conflicts of interest with respect to the authorship and/or publication of this article.

\section{Funding}

The authors received no financial support for the research and/or authorship of this article.

\section{REFERENCES}

1. Buchan A, Gates P, Pelz D, Barnett HJ. Intraluminal thrombus in the cerebral circulation. Implications for surgical management. Stroke 1988;19:681-7.

2. Vellimana AK, Kadkhodayan Y, Rich KM, Cross DT 3rd, Moran CJ, Zazulia AR, et al. Symptomatic patients with intraluminal carotid artery thrombus: outcome with a strategy of initial anticoagulation. J Neurosurg 2013;118:34-41.

3. Villarreal J, Silva J, Eliasziw M, Sharpe B, Fox A, Hachinski V, et al. North American Symptomatic Carotid Endarterectomy Trial: prognosis of patients with intraluminal thrombus in the internal carotid artery. Stroke 1998;29:276.

4. Biller J, Adams HP Jr, Boarini D, Godersky JC, Smoker WR, Kongable G. Intraluminal clot of the carotid artery. A clinical-angiographic correlation of nine patients and literature review. Surg Neurol 1986;25:467-77.

5. Walters BB, Ojemann RG, Heros RC. Emergency carotid endarterectomy. J Neurosurg 1987;66:817-23.

6. Iwata T, Mori T, Tajiri H, Miyazaki Y, Nakazaki M. Safety and effectiveness of emergency carotid artery stenting for a high-grade carotid stenosis with intraluminal thrombus under proximal flow control in hyperacute and acute stroke. J Neurointerv Surg 2013;5:40-4.

7. Sacco RL, Adams R, Albers G, Alberts MJ, Benavente O, Furie K, et al. Guidelines for prevention of stroke in patients with ischemic stroke or transient ischemic attack: a statement for healthcare professionals from the American Heart Association/American Stroke Association Council on Stroke: co-sponsored by the Council on Cardiovascular Radiology and Intervention: the American Academy of Neurology affirms the value of this guideline. Stroke 2006;37:577-617. 